\title{
Reflexiones sobre el consentimiento informado en niños, niñas y adolescentes en la atención clínica
}

\author{
Marianela Barcia*, Carlos Zunino ${ }^{\dagger}$
}

\section{Resumen}

La Convención de los Derechos del Niño ha establecido un cambio en la concepción de la infancia. Los niños, niñas y adolescentes (NNA) pasan a ser considerados sujetos de derechos y protección en lugar de objetos en la sociedad. La salud como proceso social debe acompasar este paradigma. En la relación clínica es necesario que los NNA puedan participar de la toma de decisiones que afectan su salud. El objetivo de este artículo es discutir las características particulares del consentimiento informado en NNA en el contexto de una práctica sanitaria constituida desde el paradigma propuesto por la Convención de Derechos del Niño de 1989. El consentimiento informado como expresión de voluntad para que sea válido debe cumplir tres requisitos: la competencia para tomar decisiones autónomas, la información y la libertad. El principal desafío del equipo de salud es determinar cuándo un NNA puede otorgar un consentimiento válido. En este artículo se reflexionará en torno a las características del consentimiento informado en el ámbito de la relación clínica. El equipo de salud debe garantizar la participación de los NNA en la toma de decisiones, considerando que la autonomía se adquiere gradualmente.

Palabras clave: Consentimiento informado

Niño

Adolescente

Autonomía personal

Key words: Informed consent

Child

Adolescent

Patient rights

Personal autonomy

\footnotetext{
* Doctora en Odontología. Profesora Adjunta de la Unidad Académica de Bioética. Facultad de Medicina, Universidad de la República. Asistente de la Cátedra de Odontología Social, Facultad de Odontología, Universidad de la República. Uruguay.

† Especialista en Pediatría. Profesor Adjunto de la Unidad Académica de Bioética. Facultad de Medicina, Universidad de la República. Profesor Adjunto de la Clínica Pediátrica "C", Facultad de Medicina, Universidad de la República. Uruguay.

Unidad Académica de Bioética (UAB). Facultad de Medicina. Universidad de la República. Correo electrónico: bioetica@fmed.edu.uy

Los autores declaran no tener conflicto de intereses.

Recibido: 11/2/19

Aprobado: 22/4/19
} 


\section{Introducción}

La Convención de los Derechos del Niño de 1989 plasma la voluntad de dar un giro a la concepción de la infancia y la adolescencia, considerando a niños, niñas y adolescentes (NNA) sujetos de derechos. Promueve un proceso de ciudadanía en donde se debería asegurar la libertad de información, expresión y participación en sus espacios de desarrollo y crecimiento, en las familias e instituciones, incluidos los servicios de salud ${ }^{(1)}$.

Reconocer los derechos de los NNA respecto a todas sus necesidades demanda un enfoque en el cual estos se transforman en participantes activos de sus decisiones y acciones. En el artículo 12 de esta Convención se explicita: "Los Estados Partes garantizarán al niño que esté en condiciones de formarse un juicio propio el derecho de expresar su opinión libremente en todos los asuntos que afectan al niño, teniéndose debidamente en cuenta las opiniones del niño, en función de la edad y madurez del niño" ${ }^{\text {(2) }}$. Uno de los principales desafíos es la aplicabilidad de ese artículo a la práctica sanitaria cotidiana.

En Uruguay esta Convención se aprobó por la Ley $\mathrm{N}^{\mathrm{o}} 17.823$ (Código de la niñez y adolescencia).

Sin embargo, aún persisten dificultades para que la opinión de los NNA sea tomada seriamente y se respete en temas que les involucra directa o indirectamente.

La salud como proceso social debe acompasar este paradigma. Los trabajadores de la salud deben defender, promover y garantizar los derechos de NNA. Tienen la obligación de acompañarlos en la toma de decisiones en salud. La atención a la salud se ha caracterizado por ser un espacio con una fuerte impronta paternalista principalmente para este grupo etario, aunque también para las personas adultas. La propuesta de considerar los valores y la cultura de NNA para planificar su atención implica un desafío para las instituciones y el equipo de salud. Se debe considerar la singularidad de cada NNA y su contexto, no hay un modelo universal de infancia o adolescencia. Si bien coinciden en cuanto a las edades, el enfoque debe reconocer y respetar la diversidad humana. El equipo de salud debe contar con políticas institucionales, guías de atención, herramientas profesionales y personales que permitan concretar y aplicar el enfoque de derechos a diferentes circunstancias de forma individualizada.

En la relación clínica es necesario que los NNA puedan participar de la toma de decisiones que afectan su salud. El consentimiento informado es una herramienta que permite promover la autonomía de las personas. En la infancia y la adolescencia este proceso de consentimiento informado presenta algunas particularidades específicas que generan ciertas interrogantes dentro del equipo de salud. Entre ellas: ¿qué decisiones pueden tomar los NNA relacionadas a su salud?, ¿existen límites en la toma de estas decisiones?, ¿cuándo un NNA toma decisiones autónomas?, ¿qué rol juegan los adultos referentes en la toma estas decisiones?

El objetivo de este artículo es discutir las características particulares del consentimiento informado en NNA basado en el paradigma propuesto por la Convención de Derechos del Niño de 1989.

\section{Rol de los niños, niñas y adolescentes en la toma de decisiones}

Las opiniones de los NNA deberán considerarse en todas las decisiones que los involucren, incluyendo las decisiones sobre su salud. Los NNA deberán ser escuchados e informados de sus problemas de salud de acuerdo con su nivel de comprensión, procurando que sean los protagonistas principales del proceso de atención fomentando su participación ${ }^{(3)}$.

La participación de los NNA en todos los aspectos de sus vidas, incluyendo la salud, debe ser promovida desde la primera infancia. Se pueden reconocer grados evolutivos de participación que van desde la información sobre la enfermedad, tratamiento y procedimientos médicos, toma de decisiones de los referentes adultos con participación activa de NNA, hasta decisiones autónomas tomadas por los NNA. Se deberá procurar que los NNA se vayan involucrando en la toma de decisiones en conjunto con sus referentes adultos, dado que se trata de un proceso que requiere ser aprendido y practicado con orientaciones que permitan entender las repercusiones de cada decisión. La idea de que una determinada edad cronológica por sí sola determine la autonomía de un NNA es incorrecta, sino que las variables para determinar la autonomía son individualizadas y progresivas.

En el Diccionario Latinoamericano de Bioética se define el consentimiento informado como una declaración de voluntad del paciente relativa a los procedimientos diagnósticos y terapéuticos sobre su cuerpo, tomando una decisión razonada e independiente de someterse o no a los mismos ${ }^{(4)}$. Según Pablo Simón Lorda "el consentimiento informado consiste en un proceso en el cual el médico y el paciente capaz intercambian información suficiente y comprensible acerca de la decisión sanitaria a tomar, de tal manera que se produce una dinámica activa y cooperativa de generación de esta última"(5). Se reconocen tres requisitos para que el consentimiento sea válido: la competencia, la información y libertad. Cuando se trata de NNA, el principal desafío es determinar la validez.

\section{Validez del consentimiento informado en niños, niñas y adolescentes}

Se analizarán los requisitos y consideraciones específicas que se deben tomar en cuenta para valorar cada uno 
de los aspectos que hacen que el consentimiento informado de NNA sea válido.

\section{Competencia para tomar decisiones autónomas}

En el caso de los NNA, este aspecto es el más controversial y de mayor complejidad para determinar. Es fundamental que se evalúe el grado de madurez del NNA para decidir si puede tomar determinadas decisiones con autonomía.

Históricamente se ha igualado la capacidad jurídica con la competencia de toma de decisiones. No se deben confundir estos términos, "la capacidad jurídica es la aptitud legal de una persona para la adquisición y ejercicio de sus derechos, es la idoneidad de todas las personas para ser sujeto de derecho y ejercer esos derechos". Quienes sean considerados incapaces ejercerán sus derechos y asumirán obligaciones a través de un representante legal o un curador ${ }^{(6)}$. El reconocimiento de los NNA como incapaces legales absolutos o relativos no puede constituir una barrera para que participen o tomen decisiones autónomas referidas a su salud.

Como lo explicita Galán Cortés, "cuando se trate de menores que reúnan condiciones de madurez suficiente y en los que, por tanto, su capacidad de juicio y entendimiento les permita conocer el alcance del acto médico para su propio bien jurídico, deben ser ellos mismos quienes autoricen la intervención médica" ${ }^{(7)}$.

Outomuro, citando a Culver, define competencia como "la capacidad que tiene una persona para entender $y$ apreciar la información dada durante el proceso de consentir o de rechazar una propuesta diagnóstica o terapéutica ... se refiere a una habilidad cognitiva, a la capacidad psíquica de procesar (entender, comparar y valorar) la información" (8). La capacidad cognitiva de comprender y procesar la información, junto con fundamentar la decisión y sus alternativas a través de procesos lógicos, son fundamentales para evaluar la competencia. Los NNA competentes deberán considerar las consecuencias comparando los beneficios y riesgos de sus decisiones $^{(9-11)}$.

Outomuro menciona tres características relevantes de la competencia. No se trata de una capacidad todo o nada, sino que depende del contexto y de la situación. Tampoco es una capacidad constante, de modo que una persona puede ser competente en un momento y no serlo en otro, y no toma en cuenta el tipo de decisión ${ }^{(8)}$.

El proceso de toma de decisiones variará de acuerdo con las características propias del NNA, las características del contexto y de la complejidad de las situaciones a decidir. El equipo de salud deberá valorar estas características para evaluar la competencia en cada caso. Por ejemplo, no será igual un adolescente que ha vivido con una patología crónica de larga data que un adolescente sano.

Existen diferentes instrumentos o escalas de medición con la finalidad de ayudar a los profesionales de la salud a evaluar el grado de competencia (por ejemplo, escalas de Appelbaum y Grisso, la British Medical Association \& Law Society) y el grado de severidad de la situación (por ejemplo, escala de Drane) ${ }^{(12)}$. Ninguno de estos instrumentos debe sustituir la relación clínica donde la confianza, escucha y empatía serán primordiales. La evaluación de la competencia forma parte de la asistencia y debe estar siempre presente.

Como cita Adriasola a Castellano Barca, "la evaluación de un menor para valorar su grado de madurez es una acción médica, diferente para cada caso, que variará a lo largo del tiempo y que puede estar influida por múltiples factores. Transcribo algunos criterios de madurez a juicio de Martín Espildora: comprensión adecuada de lo que se está hablando, da motivos razonables que fundamentan su petición, pondera los riesgos y beneficios de su decisión, y puede acudir solo para recibir asistencia. En ocasiones se precisará estudio psicológico" ${ }^{\text {(13) }}$. La evaluación de la competencia deberá ser realizada por el equipo de salud actuante y no exclusivamente por los profesionales de salud mental. Esto se recoge en el Decreto $N^{\circ} 274 / 2011$ que reglamenta la Ley 18.335 sobre derechos y obligaciones de los pacientes y usuarios de los servicios de salud.

\section{Información}

La información debe proporcionarse como lo indica la Ley $\mathrm{N}^{\circ}$ 18.335: "Adecuada, suficiente y continua".

El NNA o los referentes adultos deben poseer información suficiente y correcta. "El consentimiento se extiende en cuanto a su validez hasta donde haya llegado la información", (14).

El equipo de salud deberá adecuar el tipo de lenguaje a las características de cada NNA. La edad per se no es una limitante para recibir información sobre cualquier aspecto de su salud. La cantidad de información recibida deberá ser gradual, de acuerdo con la posibilidad de comprensión y a los requerimientos individuales, procurando incluir a los adultos referentes ${ }^{(15)}$. Se debe tener en cuenta que "la información debe ser trasladada al paciente con la antelación suficiente para que pueda reflexionar sobre su decisión" ${ }^{(7)}$. El equipo de salud deberá favorecer instancias de reflexión y de asesoramiento para la toma de decisiones. Dar información a un NNA es reconocerlo como sujeto de derecho y es independiente de que la decisión final sea tomada por sí solo.

Informar al NNA y fomentar su implicación en la toma de decisiones favorece diversos aspectos en el proceso salud/enfermedad. Aumenta la satisfacción de los 
cuidados recibidos, favorece la incorporación de hábitos saludables, promoción de salud y prevención de enfermedades, adherencia a los tratamientos y la continuidad asistencial. Además, genera empoderamiento de los aspectos relacionados a su salud ${ }^{(3)}$.

\section{Libertad}

La libertad para consentir se relaciona a la ausencia de coacción, elemento que se debe considerar en general en la práctica asistencial que de por sí es una práctica asimétrica, que se evidencia más aún cuando el paciente es un NNA.

La presencia de los adultos referentes identificados por los NNA podría ser una guía y apoyo de estos, pero no debe convertirse en un elemento de coacción. No se trata de excluir a los adultos de los procesos de toma de decisiones, sino de que participen respetando las opiniones y necesidades de cada NNA. Se deben considerar situaciones no explícitas que puedan estar coaccionando la toma de decisiones, como la violencia intrafamiliar en cualquiera de sus dimensiones, falta de autonomía económica, entre otras.

De los tres elementos analizados que determinan la validez del consentimiento informado, la evaluación de la competencia es el que genera más conflicto. Sin embargo, esto no debe dejar de lado el análisis de los puntos de la información y la libertad. Los últimos, en su mayor medida, dependen de los adultos que intervienen en la situación. Los profesionales de la salud tienen un compromiso en promover la actitud participativa de los NNA reconociéndolos como verdaderos protagonistas del proceso de atención y dándoles la información con las características desarrolladas, asegurándose que la decisión se tome con libertad.

Se debe tener en cuenta que en nuestro país se reconocen el derecho a la información y el acceso a los servicios de salud, y el concepto de autonomía progresiva en la Ley $\mathrm{N}^{\circ} 18.426$, que en su artículo 7 agrega al Código de la niñez y la adolescencia el artículo 11 bis estableciendo: "Todo niño, niña o adolescente tiene derecho a la información y acceso a los servicios de salud, inclusive los referidos a la salud sexual y reproductiva, debiendo los profesionales actuantes respetar la confidencialidad de la consulta y ofrecerle las mejores formas de atención y tratamiento cuando corresponda. De acuerdo a la edad del niño, niña o adolescente se propenderá a que las decisiones sobre métodos de prevención de la salud sexual u otros tratamientos médicos que pudieran corresponder, se adopten en concurrencia con sus padres u otros referentes adultos de su confianza, debiendo respetarse en todo caso la autonomía progresiva de los adolescentes. En caso de existir riesgo grave para la salud del niño, niña o adolescente y no pudiera llegarse a un acuerdo con éste o con sus padres o responsables del mismo en cuanto al tratamiento a seguir, el profesional podrá solicitar el aval del Juez competente en materia de derechos vulnerados o amenazados de niños, niñas y adolescentes, quien a tales efectos deberá recabar la opinión del niño, niña o adolescente, siempre que sea posible".

Desafíos para el respeto de la autonomía de niños, niñas $y$ adolescentes

Se deben considerar los posibles conflictos y diferentes opiniones entre los adultos referentes y los NNA. Es de suma importancia aceptar que se trata de un proceso donde el diálogo y el encuentro de acuerdos es clave y muchas veces no inmediato, pero debe estar orientado por el respeto del principio de autonomía y en casos de NNA no competentes lograr su mayor participación, contemplando su interés superior.

Para lograr que los NNA puedan ejercer sus derechos en los servicios de salud es de suma importancia la sensibilización y formación continua del equipo de salud. Se debe continuar con el proceso de incorporación de la temática en los currículos de formación o capacitación de todos los trabajadores de la salud.

\section{Conclusiones}

La promoción y el respeto de la autonomía de los NNA forma parte del modelo de atención basado en derechos y es responsabilidad de todo el equipo de salud garantizarlo. Se debe procurar la toma de decisiones desde la infancia logrando de forma gradual la autonomía.

El rol que le atribuimos a los NNA en este artículo de la atención sanitaria no puede ser pensado de forma aislada, sino que debe ser articulado con un modelo de infancia y adolescencia en un contexto de responsabilidad en la vida adulta.

\section{Agradecimientos}

Prof. Dr. Hugo Rodríguez Almada.

\section{Abstract}

The Convention on the Rights of the Child has established a change in the concept of childhood.

Children become subjects of rights and protection instead of being objects of society. The health contexts, as a sphere for social processes needs to keep pace with this paradigm. It is necessary for children and adolescents to participate in the making of decisions affecting their health in the clinical context. The study aims to discuss the specific characteristics of informed consent in children and adolescents within the context of the provision of healthcare services from the paradigm proposed by the Convention of the Rights of the Child of 1989. Informed 
consent as an expression of will, needs to meet three requirements to be valid: competence to make autonomous decisions, information and freedom. The main challenge of the health team lies in defining the moment when a child and adolescent may grant a valid consent. This study will reflect on the characteristics of informed consent within the context of the provision of health care services. The health team must guarantee the participation of children and adolescents in the making of decisions, understanding autonomy is gradually acquired.

\section{Resumo}

A Convenção dos Direitos da Criança estabeleceu uma modificação do conceito de infância. Os meninos, meninas e adolescentes (NNA) passam a ser considerados sujeitos de direito e proteção e não como objetos na sociedade. A saúde como processo social deve acompanhar este paradigma. Na relação clínica é necessário que os NNA possam participar da tomada de decisões quando estas afetam sua saúde. O objetivo deste artigo é discutir as características particulares do consentimento informado em meninos, meninas e adolescentes no contexto de uma prática sanitária constituída a partir do paradigma proposto pela Convenção dos Direitos da Criança de 1989. Para que o consentimento informado como expressão de vontade seja válido deve cumprir com três requisitos: a competência para tomar decisões autônomas, a informação e a liberdade. O principal desafio para a equipe de saúde é determinar quando um NNA pode outorgar um consentimento válido. Neste artigo faz-se uma reflexão sobre as características do consentimento informado no âmbito da relação clínica. A equipe de saúde deve garantir a participação dos NNA na tomada de decisões, considerando que a autonomia é adquirida de forma gradual.

\section{Bibliografía}

1. Ministerio de Salud Pública, UNICEF, INAU, UdelaR, SUP. Guía: los derechos de niños, niñas y adolescentes en el área de salud. Montevideo: UNICEF, 2012.
2. ACNUDH. Convención sobre los Derechos del Niño, 1989. Disponible en: www.ohchr. org/spanish/law/crc.htm. [Consulta: 12 nov. 2018].

3. Esquerda Aresté M, Pifarré Paradero J, Miquel Fernández E. La capacidad de decisión en el menor. Aspectos particulares de la información en el niño y en el joven. An Pediatr Contin. 2013; 11(4):204-11.

4. Wierzba S. Consentimiento: información En: Tealdi JC, dir. Diccionario Latinoamericano de Bioética. Bogotá: UNESCO-Red Latinoamericana y del Caribe de Bioética: Universidad Nacional de Colombia, 2008:216-7.

5. Simón Lorda P. El consentimiento informado y la participación del enfermo en las relaciones sanitarias. MEDIFAM 1995; 5(5):264-71.

6. Gamarra J. Tratado de Derecho Civil Uruguayo $4^{\mathrm{a}}$ ed. Montevideo: FCU, 2013:40-56, tomo X.

7. Galán Cortés JC. Responsabilidad médica y el consentimiento informado. Madrid: Civitas, 2001:78-91.

8. Outomuro D. Manual de fundamentos de Bioética. Buenos Aires: Magíster EOS, 2004:6-97.

9. Rueda L. Consentimiento informado en niños y adolescentes. ARS Médica 2008; 37(1):1-15.

10. Appelbaum P, Grisso T. Assessing patients' capacities to consent to treatment. N Engl J Med 1988; 319:1635-8.

11. Appelbaum P. Clinical practice. Assessment of patients' competence to consent to treatment. N Engl J Med 2007; 357(18):1834-40.

12. Esquerda M, Pifarré J, Miquel E. El consentimiento informado en adolescentes: aspectos legales. En: Hidalgo M, Redondo A, Castellano G. Medicina de la Adolescencia: atención integral. Barcelona: Océano, 2012:29-34.

13. Adriasola G. Aproximación al secreto médico del adolescente. Rev Méd Urug 2008; 24(3):212-21.

14. González D, Rodríguez H, Berro R. Consentimiento informado: análisis crítico de su aplicación en un servicio quirúrgico. Rev Méd Urug 2005; 21(4):291-7.

15. Rodríguez H. Consentimiento informado en la práctica clínica. AnFaMed 2017(4):22-30. 